\title{
Compatible spreads of symmetry in near polygons
}

\author{
Bart De Bruyn
}

Received: July 23, 2004 / Revised: April 1, 2005 / Accepted: July 8, 2005

(C) Springer Science + Business Media, Inc. 2006

\begin{abstract}
In De Bruyn [7] it was shown that spreads of symmetry of near polygons give rise to many other near polygons, the so-called glued near polygons. In the present paper we will study spreads of symmetry in product and glued near polygons. Spreads of symmetry in product near polygons do not lead to new glued near polygons. The study of spreads of symmetry in glued near polygons gives rise to the notion of 'compatible spreads of symmetry'. We will classify all pairs of compatible spreads of symmetry for the known classes of dense near polygons. All these pairs of spreads can be used to construct new glued near polygons.
\end{abstract}

Keywords Near polygon $\cdot$ Generalized quadrangle $\cdot$ Spread

\section{Elementary definitions}

A near polygon [19] is a partial linear space $\mathcal{S}=(\mathcal{P}, \mathcal{L}, \mathrm{I}), \mathrm{I} \subseteq \mathcal{P} \times \mathcal{L}$, with the property that for every point $p \in \mathcal{P}$ and every line $L \in \mathcal{L}$ there exists a unique point on $L$ nearest to $p$. Here distances $\mathrm{d}(\cdot, \cdot)$ are measured in the point graph or collinearity graph $\Gamma$. If $d=\operatorname{diam}(\mathcal{S})$ denotes the diameter of $\Gamma$ (or of $\mathcal{S}$ ), then the near polygon is called a near $2 d$-gon. A near 0 -gon is a point and a near 2-gon is a line.

If $X_{1}$ and $X_{2}$ are two sets of points, then $\mathrm{d}\left(X_{1}, X_{2}\right)$ denotes the minimal distance between a point of $X_{1}$ and a point of $X_{2}$. If $X_{1}=\{x\}$, then we also write $\mathrm{d}\left(x, X_{2}\right)$ instead of $\mathrm{d}\left(\{x\}, X_{2}\right)$. For every $i \in \mathbb{N}, \Gamma_{i}\left(X_{1}\right)$ denotes the set of all points $y$ for which $\mathrm{d}\left(y, X_{1}\right)=i$. If $X_{1}=\{x\}$, we also write $\Gamma_{i}(x)$ instead of $\Gamma_{i}(\{x\})$.

A near $2 d$-gon, $d \geq 2$, is called a generalized $2 d$-gon [20] if $\left|\Gamma_{i-1}(x) \cap \Gamma_{1}(y)\right|=1$ for every $i \in\{1, \ldots, d-1\}$ and every two points $x$ and $y$ at distance $i$ from each other. A generalized $2 d$-gon is called degenerate if it does not contain ordinary $2 d$-gons as

Postdoctoral Fellow of the Research Foundation-Flanders.

B. D. Bruyn $(\square)$

Department of Pure Mathematics and Computer Algebra, Ghent University, Galglaan 2,

B-9000 Gent, Belgium

e-mail: bdb@cage.Ugent.be 
subgeometries, or equivalently, if it contains a point which has distance at most $d-1$ from any other point. The near quadrangles are precisely the generalized quadrangles (GQ's, [18]). A degenerate generalized quadrangle consists of a number of lines through a point.

Let $X$ be a nonempty set of points of a near polygon $\mathcal{S}$. The set $X$ is called a subspace if every line meeting $X$ in at least two points is completely contained in $X$. The set $X$ is called geodetically closed if it is a subspace and if every point on a shortest path between two points of $X$ is as well contained in $X$. If $X$ is a subspace, then we can define a subgeometry $\mathcal{S}_{X}$ of $\mathcal{S}$ by considering only those points and lines of $\mathcal{S}$ which are completely contained in $X$. If $X$ is geodetically closed, then $\mathcal{S}_{X}$ clearly is a sub near polygon of $\mathcal{S}$. If $\mathcal{S}_{X}$ is a nondegenerate generalized quadrangle, then $X$ and often also $\mathcal{S}_{X}$ will be called a quad. If $X_{1}, \ldots, X_{k}$ are nonempty sets of points, then $\mathcal{C}\left(X_{1}, \ldots, X_{k}\right)$ denotes the minimal geodetically closed sub near polygon through $X_{1} \cup \cdots \cup X_{k}$, i.e. the intersection of all geodetically closed sub near polygons through $X_{1} \cup \cdots \cup X_{k}$. If $x$ and $y$ are two different points of $\mathcal{S}$, then we denote $\mathcal{C}(\{x, y\})$ also by $\mathcal{C}(x, y)$.

A near polygon is said to have $\operatorname{order}(s, t)$ if every line is incident with exactly $s+1$ points and if every point is incident with exactly $t+1$ lines. A near polygon is called thin if every line is incident with precisely two points. The thin near polygons are precisely the bipartite graphs (if one regards the edges as lines). A near polygon is called dense if every line is incident with at least three points and if every two points at distance 2 have at least two common neighbours. Dense near polygons satisfy several nice properties. By Lemma 19 of [2], every point of a dense near polygon $\mathcal{S}$ is incident with the same number of lines. We denote this number by $t_{\mathcal{S}}+1$. If $x$ and $y$ are two points of a dense near polygon at distance $\delta$ from each other, then by Theorem 4 of [2], there exists a unique geodetically closed sub near $2 \delta$-gon through $x$ and $y$ which necessarily coincides with $\mathcal{C}(x, y)$. So, if $x$ and $y$ are two points at distance 2 in a dense near polygon, then these points are contained in a unique quad.

A geodetically closed sub near polygon $F$ of a near polygon $\mathcal{S}$ is called classical if for every point $x$ there exists a (necessarily unique) point $\pi_{F}(x)$ in $F$ such that $\mathrm{d}(x, y)=$ $\mathrm{d}\left(x, \pi_{F}(x)\right)+\mathrm{d}\left(\pi_{F}(x), y\right)$ for every point $y$ of $F$. Obviously, every line of a near polygon is classical. If $F_{1}$ and $F_{2}$ denote two classical sub near polygons, then we denote by $\pi_{F_{1}, F_{2}}$ the restriction of $\pi_{F_{2}}$ to the point set of $F_{1}$. Two classical sub near polygons $F_{1}$ and $F_{2}$ are called parallel if $\pi_{F_{1}, F_{2}}$ is an isomorphism. If this is the case, then also $\pi_{F_{2}, F_{1}}$ is an isomorphism and $\pi_{F_{2}, F_{1}}^{-1}=\pi_{F_{1}, F_{2}}$. The parallel relation is not necessarily transitive. We denote the set of all partitions of $\mathcal{S}$ in mutually parallel classical geodetically closed sub near polygons by $\Upsilon(\mathcal{S})$.

A spread of a near polygon $\mathcal{S}$ is a set of lines partitioning the point set. A spread is called admissible if it belongs to $\Upsilon(\mathcal{S})$, or equivalently, if every two lines of it are parallel. Obviously, every spread of a generalized quadrangle is admissible. A spread $S$ is called regular if it is admissible and if the following holds for any two lines $K, L \in S$ with $\mathrm{d}(K, L)=1$ : (i) $\{K, L\}^{\perp \perp}$ and $\{K, L\}^{\perp}$ cover the same set of points of $\mathcal{S}$, (ii) every line of $\{K, L\}^{\perp \perp}$ belongs to $S$. $\left(\{K, L\}^{\perp}\right.$ is the set of lines of $\mathcal{S}$ meeting $K$ and $L,\{K, L\}^{\perp \perp}$ is the set of lines of $\mathcal{S}$ meeting each line of $\{K, L\}^{\perp}$.) A spread $S$ is called a spread of symmetry if for every line $K \in S$ and for every two points $k_{1}$ and $k_{2}$ on $K$ there exists an automorphism of $\mathcal{S}$ fixing each line of $S$ and mapping $k_{1}$ to $k_{2}$. Obviously, every spread of symmetry is regular. If $S$ is an admissible spread (a spread of symmetry) of a near polygon $\mathcal{S}$ and if $F$ is a geodetically closed sub near polygon of $\mathcal{S}$, then the set $S_{F}$ of all lines of $S$ which are contained in $F$ is either empty or an admissible spread (a spread of symmetry) of $F$, see e.g. Theorem 5 of [7]. 


\section{Motivation and short overview}

Spreads of symmetry give rise to new near polygons, the so-called glued near polygons, see [5] and [7]. For all known classes of indecomposable dense near polygons (these are dense near polygons which are not glued and not a product near polygon), all spreads of symmetry have been determined (see Section 5 for an overview). Something which has not yet been done is the study of spreads of symmetry in glued near polygons themselves. This study, see Theorems 4.4 and 4.5 , led to the notion of compatible spreads of symmetry which we will discuss in Section 3 . The known examples of compatible spreads of symmetry of indecomposable dense near polygons are listed in Section 5. Each such pair of spreads will give rise to new glued near polygons. In Theorem 4.7, we will describe how compatible spreads of symmetry in glued near polygons are obtained. This theorem can be used to construct further examples of glued near polygons. In order to be complete, we also study (compatible) spreads of symmetry in product near polygons (Theorems 4.4, 4.5 and 4.6), but this will essentially not lead to new glued near polygons.

\section{Compatible spreads of symmetry}

Theorem 3.1. Let $\mathcal{A}=(\mathcal{P}, \mathcal{L}, \mathrm{I})$ be a near polygon, let $S_{1}$ and $S_{2}$ denote two different spreads of symmetry in $\mathcal{A}$ and let $G_{i}, i \in\{1,2\}$, denote the group of automorphisms of $\mathcal{A}$ which fix each line of $S_{i}$. Then the following are equivalent:

(i) $\left[G_{1}, G_{2}\right]=0$;

(ii) for every line $l \in S_{1}$ and every $g \in G_{2}, l^{g} \in S_{1}$;

(iii) for every line $l \in S_{2}$ and every $g \in G_{1}, l^{g} \in S_{2}$;

(iv) the partial linear space $\mathcal{B}=\left(\mathcal{P}, S_{1} \cup S_{2}, \mathrm{I}_{\mid \mathcal{P} \times\left(S_{1} \cup S_{2}\right)}\right)$ is a disjoint union of lines and grids.

Proof: $(i) \Rightarrow($ ii $)$ and $(i) \Rightarrow($ iii $)$ : By symmetry, it suffices to prove the implication $(i) \Rightarrow$ (ii). Let $l$ denote an arbitrary line of $S_{1}$, let $x$ denote an arbitrary point of $l$ and let $g$ denote an arbitrary element of $G_{2}$. Then $l^{g}=\left(x^{G_{1}}\right)^{g}=\left(x^{g}\right)^{G_{1}} \in S_{1}$.

$(i i) \Rightarrow(i v)$ and $(i i i) \Rightarrow(i v)$ : By symmetry, it suffices to prove the implication $(i i) \Rightarrow(i v)$. Suppose that the lines $K_{1} \in S_{1}$ and $K_{2} \in S_{2}$ intersect in a point $x^{*}$. For all $x_{1} \in K_{1}$, $x_{1}^{G_{2}} \in S_{2}$ and for all $x_{2} \in K_{2}, x_{2}^{G_{1}} \in S_{1}$. We will now prove that the lines $x_{1}^{G_{2}}, x_{1} \in K_{1}$, and $x_{2}^{G_{1}}, x_{2} \in K_{2}$, define a subgrid of $\mathcal{B}$. Obviously, $x_{1}^{G_{2}} \cap x_{1}^{\prime G_{2}}=\emptyset$ for all $x_{1}, x_{1}^{\prime} \in K_{1}$ with $x_{1} \neq x_{1}^{\prime}$ and $x_{2}^{G_{1}} \cap x_{2}^{\prime G_{1}}=\emptyset$ for all $x_{2}, x_{2}^{\prime} \in K_{2}$ with $x_{2} \neq x_{2}^{\prime}$. Now, consider arbitrary points $x_{1} \in K_{1}$ and $x_{2} \in K_{2}$ and let $g_{2}$ denote an arbitrary element of $G_{2}$ such that $x_{2}=\left(x^{*}\right)^{g_{2}}$. The point $x_{2}$ lies on the line $K_{1}^{g_{2}}$ which, by our assumption, belongs to the spread $S_{1}$. So, $x_{2}^{G_{1}}=K_{1}^{g_{2}}$ and $x_{2}^{G_{1}} \cap x_{1}^{G_{2}}=K_{1}^{g_{2}} \cap x_{1}^{G_{2}}=\left\{x_{1}^{g_{2}}\right\}$. As a consequence, every two different intersecting lines of $S_{1} \cup S_{2}$ are contained in a subgrid of $\mathcal{B}$. The implication now follows from the fact that every point of $\mathcal{B}$ is contained in at most two lines of $\mathcal{B}$.

(iv) $\Rightarrow(i)$ : Let $x$ be an arbitrary point of $\mathcal{A}$, let $g_{1}$ be an arbitrary element of $G_{1}$ and let $g_{2}$ be an arbitrary element of $G_{2}$. We will prove that $x^{g_{1} g_{2}}=x^{g_{2} g_{1}}$. We distinguish the following cases. 
- Suppose that $x$ is contained in a subgrid $G$ of $\mathcal{B}$. Let $l_{i}, i \in\{1,2\}$, denote the unique line of $S_{i}$ through $x$. Since $x^{g_{i}} \in l_{i}$, the unique line $m_{3-i}$ of $S_{3-i}$ through $x^{g_{i}}$ is contained in $G$. Let $y$ be the common point of the lines $m_{1}$ and $m_{2}$. Since $x \sim x^{g_{1}}, x^{g_{2}} \sim x^{g_{1} g_{2}}$. So, $x^{g_{1} g_{2}}$ is the unique point of $m_{2}$ collinear with $x^{g_{2}}$. Hence, $y=x^{g_{1} g_{2}}$. In a similar way, one proves that $y=x^{g_{2} g_{1}}$. As a consequence, $x^{g_{1} g_{2}}=x^{g_{2} g_{1}}$.

- Suppose that $x$ is not contained in a subgrid of $\mathcal{B}$, i.e. $x$ is contained in a line $L$ of $S_{1} \cap S_{2}$. Since $S_{1} \neq S_{2}, \mathcal{B}$ has a subgrid $G$. Every line of $G$ is parallel with $L$. Let $y \in G$ such that $x$ is the unique point of $L$ nearest to $x$. Then $x^{g_{1} g_{2}}$ (respectively $x^{g_{2} g_{1}}$ ) is the unique point of $L=L^{g_{1} g_{2}}=L^{g_{2} g_{1}}$ nearest to $y^{g_{1} g_{2}}$ (respectively $y^{g_{2} g_{1}}$ ). Since $y^{g_{1} g_{2}}=y^{g_{2} g_{1}}$, it follows that $x^{g_{1} g_{2}}=x^{g_{2} g_{1}}$.

Definition. Let $\mathcal{A}$ be a near polygon, let $S_{1}$ and $S_{2}$ be two (possibly equal) spreads of symmetry of $\mathcal{A}$ and let $G_{i}, i \in\{1,2\}$, denote the group of automorphisms of $\mathcal{A}$ which fix each line of $S_{i}$. Then the spreads $S_{1}$ and $S_{2}$ are called compatible if $\left[G_{1}, G_{2}\right]=0$. In the case that $S_{1}$ and $S_{2}$ are different, Theorem 3.1 allows us to give some equivalent definitions.

\section{Importance of compatible spreads of symmetry}

In this section we will show that compatible spreads of symmetry give rise to spreads of symmetry in glued near polygons and hence also to new glued near polygons.

\subsection{Product and glued near polygons}

Let $\mathcal{A}$ be a dense near polygon. For every $i \in\{0,1\}$, let $\Delta_{i}(\mathcal{A})$ denote the set of all pairs $\left\{T_{1}, T_{2}\right\}$ satisfying the following properties:

(1) $T_{j}, j \in\{1,2\}$, is a partition of $\mathcal{A}$ in geodetically closed sub near polygons of diameter at least $i+1$;

(2) every element of $T_{1}$ intersects every element of $T_{2}$ in a point (if $i=0$ ) or a line (if $i=1$ );

(3) every line of $\mathcal{A}$ is contained in at least one element of $T_{1} \cup T_{2}$;

(4) if $i=1$, then the spread induced in every element $F \in T_{j}, j \in\{1,2\}$, by intersecting it with the elements of $T_{3-j}$ is an admissible spread.

If $\left\{T_{1}, T_{2}\right\} \in \Delta_{i}(\mathcal{A})$, then by Lemma 2 of [11] and Corollary 4.7 of [9], there exist near polygons $\mathcal{A}_{1}$ and $\mathcal{A}_{2}$ such that every element of $T_{j}, j \in\{1,2\}$, is isomorphic to $\mathcal{A}_{j}$. If $j=0$, then $\mathcal{A}$ is the product near polygon $\mathcal{A}_{1} \times \mathcal{A}_{2}$. If $i=1$, then $\mathcal{A}$ is a so-called glued near polygon and we will say that $\mathcal{A}$ is a glued near polygon of type $\mathcal{A}_{1} \otimes \mathcal{A}_{2}$. We refer to [7] for the precise definition of glued near polygon. A spread $S$ of $\mathcal{A}$ is said to be trivial if there exists a $T \in \Upsilon(\mathcal{A})$ such that $\{S, T\} \in \Delta_{0}(\mathcal{A})$. If $\left\{T_{1}, T_{2}\right\} \in \Delta_{1}(\mathcal{A}) \backslash \Delta_{0}(\mathcal{A})$, then the spread induced in every element $F \in T_{j}, j \in\{1,2\}$, by intersecting it with the elements of $T_{3-j}$ is a spread of symmetry. So, glued near polygons give rise to spreads of symmetry. Also the converse is true. If $S_{1}$ and $S_{2}$ are spreads of symmetry of near polygons $\mathcal{A}_{1}$ and $\mathcal{A}_{2}$ satisfying certain properties (see Theorem 14 of [7]), then they give rise to glued near polygons of type $\mathcal{A}_{1} \otimes \mathcal{A}_{2}$. In this paper we study spreads of symmetry in product and glued near polygons. Spreads of symmetry in glued near polygons will give rise to new glued near polygons. Spreads of symmetry in product near polygons will essentially not give rise to new near polygons, because by Lemma 8 of [13], every near polygon of type $\left(\mathcal{A}_{1} \times \mathcal{A}_{2}\right) \otimes \mathcal{A}_{3}$ is also of type $\left(\mathcal{A}_{j} \otimes \mathcal{A}_{3}\right) \times \mathcal{A}_{3-j}$ for a certain $j \in\{1,2\}$. 


\subsection{The sets $\Upsilon_{0}(\mathcal{A})$ and $\Upsilon_{1}(\mathcal{A})$ for a dense near polygon $\mathcal{A}$}

Let $\mathcal{A}$ be a dense near polygon. We say that an element $T \in \Upsilon(\mathcal{A})$ belongs to $\Upsilon_{i}(\mathcal{A}), i \in\{0,1\}$, if there exists a $T^{\prime} \in \Upsilon(\mathcal{A})$ such that $\left\{T, T^{\prime}\right\} \in \Delta_{i}(\mathcal{A})$.

Proposition 4.1. If $T \in \Upsilon_{0}(\mathcal{A})$, then there exists a unique $T^{\prime} \in \Upsilon(\mathcal{A})$ such that $\left\{T, T^{\prime}\right\} \in$ $\Delta_{0}(\mathcal{A})$.

Proof: Let $x$ denote an arbitrary point of $\mathcal{A}$, let $F$ denote the unique element of $T$ through $x$ and let $L_{1}, \ldots, L_{k}$ denote all the lines through $x$ not contained in $T$. If $T^{\prime}$ is an element of $\Upsilon(\mathcal{A})$ such that $\left\{T, T^{\prime}\right\} \in \Delta_{0}(\mathcal{A})$, then the unique element of $T^{\prime}$ through $x$ coincides with $\mathcal{C}\left(L_{1}, \ldots, L_{k}\right)$. This proves the proposition.

Proposition 4.2. If $T \in \Upsilon_{1}(\mathcal{A}) \backslash \Upsilon_{0}(\mathcal{A})$, then there exists a unique $T^{\prime} \in \Upsilon(\mathcal{A})$ such that $\left\{T, T^{\prime}\right\} \in \Delta_{1}(\mathcal{A})$.

Proof: Let $x$ denote an arbitrary point of $\mathcal{S}$, let $F_{x}$ denote the unique element of $T$ through $x$ and let $L_{1}, \ldots, L_{k}$ denote the lines through $x$ not contained in $F_{x}$. Let $T^{\prime}$ be an element of $\Upsilon(\mathcal{A})$ such that $\left\{T, T^{\prime}\right\} \in \Delta_{1}(\mathcal{A})$ and let $F_{x}^{\prime}$ denote the unique element of $T^{\prime}$ through $x$. Clearly, $\operatorname{diam}\left(F_{x}\right)+\operatorname{diam}\left(F_{x}^{\prime}\right)=\operatorname{diam}(\mathcal{A})+1, \operatorname{diam}\left(F_{x} \cap F_{x}^{\prime}\right)=1$ and $L_{1}, \ldots, L_{k} \subseteq F_{x}^{\prime}$. There are two possibilities.

(a) $\mathcal{C}\left(L_{1}, \ldots, L_{k}\right) \cap F_{x}=\{x\}$.

In this case, we have $\operatorname{diam}\left(\mathcal{C}\left(L_{1}, \ldots, L_{k}\right)\right)=\operatorname{diam}\left(F_{x}^{\prime}\right)-1$ and hence $\operatorname{diam}(\mathcal{A})=$ $\operatorname{diam}\left(F_{x}\right)+\operatorname{diam}\left(\mathcal{C}\left(L_{1}, \ldots, L_{k}\right)\right)$. By Lemma 3 of [11], $T \in \Upsilon_{0}(\mathcal{A})$, a contradiction.

(b) $\mathcal{C}\left(L_{1}, \ldots, L_{k}\right) \cap F_{x}$ is a line.

In this case, we have $\mathcal{C}\left(L_{1}, \ldots, L_{k}\right)=F_{x}^{\prime}$.

The proposition now easily follows.

Remark. The previous proposition is not necessarily valid if $T \in \Upsilon_{0}(\mathcal{A})$. Suppose that $\left\{T, T^{\prime}\right\} \in \Delta_{0}(\mathcal{A})$, let $F \in T$ and let $S$ be an admissible spread of $F$. For every point $x$ of $F$, define $\tilde{F}_{x}:=\mathcal{C}\left(L_{x}, F_{x}^{\prime}\right)$, where $L_{x}$ denotes the unique line of $S$ through $x$ and $F_{x}^{\prime}$ denotes the unique element of $T^{\prime}$ through $x$. Put $\tilde{T}:=\left\{\tilde{F}_{x} \mid x \in F\right\}$. Then $\{T, \tilde{T}\} \in \Delta_{1}(\mathcal{A})$. So, if the near polygon $F$ has two different admissible spreads, there exists at least two $\tilde{T} \in \Upsilon(\mathcal{A})$ such that $\{T, \tilde{T}\} \in \Delta_{1}(\mathcal{A})$.

Definitions. Let $T \in \Upsilon_{0}(\mathcal{A}) \cup \Upsilon_{1}(\mathcal{A})$. If $T \in \Upsilon_{0}(\mathcal{A})$, then we denote by $T^{C}$ the unique element of $\Upsilon_{0}(\mathcal{A})$ such that $\left\{T, T^{C}\right\} \in \Delta_{0}(\mathcal{A})$. If $T \in \Upsilon_{1}(\mathcal{A}) \backslash \Upsilon_{0}(\mathcal{A})$, then we denote by $T^{C}$ the unique element of $\Upsilon(\mathcal{A})$ such that $\left\{T, T^{C}\right\} \in \Delta_{1}(\mathcal{A})$. We call $T^{C}$ the complementary partition of $T$. If $T \in \Upsilon_{0}(\mathcal{A})$, then $\left(T^{C}\right)^{C}=T$. If $T \in \Upsilon_{1}(\mathcal{A}) \backslash \Upsilon_{0}(\mathcal{A})$, then $\left(T^{C}\right)^{C}$ is not necessarily equal to $T$ (see the previous remark). We denote by $\tilde{\Upsilon}_{1}(\mathcal{A})$ the set of all $T \in \Upsilon_{1}(\mathcal{A}) \backslash \Upsilon_{0}(\mathcal{A})$ for which $\left(T^{C}\right)^{C}=T$. We also define $\tilde{\Delta}_{1}(\mathcal{A}):=\Delta_{1}(\mathcal{A}) \cap\left(\begin{array}{c}\tilde{\Upsilon}_{1}(\mathcal{A}) \\ 2\end{array}\right)$.

\subsection{Extensions of spreads and automorphisms}

Let $\mathcal{A}$ be a dense near polygon, let $T$ denote an element of $\Upsilon_{0}(\mathcal{A}) \cup \tilde{\Upsilon}_{1}(\mathcal{A})$, let $F$ denote an arbitrary element of $T$ and let $F^{\prime}$ denote an arbitrary element of $T^{C}$. 
- For every spread $S$ of $F$, we define $\bar{S}:=\left\{\pi_{F, E}(L) \mid E \in T\right.$ and $\left.L \in S\right\}$. Obviously, $\bar{S}$ is a spread of $\mathcal{A}$. We call $\bar{S}$ the extension of $S$.

- For every automorphism $\theta$ of $F$ and for every point $x$ of $\mathcal{A}$, we define $\bar{\theta}(x):=\pi_{F, F_{x}} \circ \theta \circ$ $\pi_{F_{x}, F}(x)$. Here $F_{x}$ denotes the unique element of $T$ through $x$. Obviously, $\bar{\theta}$ is a permutation of the point set of $\mathcal{A}$. We call $\bar{\theta}$ the extension of $\theta$.

Proposition 4.3. (a) Let $T \in \Upsilon_{0}(\mathcal{A})$.

(a1) If $\theta$ is an automorphism of $F$, then $\bar{\theta}$ is an automorphism of $\mathcal{A}$.

(a2) If $\phi$ is an automorphism of $\mathcal{A}$ fixing each element of $T$, then $\phi=\bar{\theta}$ for some automorphism $\theta$ of $F$.

(a3) If $\phi_{1}$ is an automorphism of $\mathcal{A}$ fixing each element of $T$ and if $\phi_{2}$ is an automorphism of $\mathcal{A}$ fixing each element of $T^{C}$, then $\phi_{1}$ and $\phi_{2}$ commute.

(b) Let $T \in \tilde{\Upsilon}_{1}(\mathcal{A})$, let $S^{*}$ denote the spread of $F$ obtained by intersecting $F$ with every element of $T^{C}$ and let $G^{*}$ denote the group of automorphisms of $F$ fixing each line of $S^{*}$. Let $\theta$ denote an automorphism of $F$. Then $\bar{\theta}$ is an automorphism of $\mathcal{A}$ if and only if $\theta$ commutes with every element of $G^{*}$.

Proof: Properties (a1), (a2) and (a3) are straightforward. In order to prove property (b), it suffices to prove that $\bar{\theta}$ maps collinear points $x$ and $y$ to collinear points $\bar{\theta}(x)$ and $\bar{\theta}(y)$. There are two possibilities.

- $F_{x}=F_{y}$.

The statement follows from the fact that the maps $\pi_{F_{x}, F}, \theta$ and $\pi_{F, F_{x}}$ are isomorphisms.

- $F_{x} \neq F_{y}$.

The points $\bar{\theta}(x)$ and $\bar{\theta}(y)$ are collinear if and only if $\pi_{F_{x}, F_{y}} \circ \bar{\theta}(x)=\bar{\theta}(y)$, i.e. if and only if $\pi_{F_{x}, F_{y}} \circ \pi_{F, F_{x}} \circ \theta \circ \pi_{F_{x}, F}(x)=\pi_{F, F_{y}} \circ \theta \circ \pi_{F_{y}, F} \circ \pi_{F_{x}, F_{y}}(x)$.

Hence, $\bar{\theta}$ is an automorphism if and only if $\pi_{F_{x}, F_{y}} \circ \pi_{F, F_{x}} \circ \theta=\pi_{F, F_{y}} \circ \theta \circ \pi_{F_{y}, F} \circ \pi_{F_{x}, F_{y}} \circ$ $\pi_{F, F_{x}}$ for all $F_{x}, F_{y} \in T$, i.e. if and only if $\theta$ commutes with $\pi_{F_{y}, F} \circ \pi_{F_{x}, F_{y}} \circ \pi_{F, F_{x}}$ for all $F_{x}, F_{y} \in T$. Let $S^{\prime}$ denote the spread of $F^{\prime}$ obtained by intersecting $F^{\prime}$ with every element of $T$. Since $T \in \tilde{\Upsilon}_{1}(\mathcal{A}), S^{\prime}$ is not a trivial spread of $F^{\prime}$ and $S^{*}$ is not a trivial spread of $F$. Put $H:=\left\{\pi_{F_{y}, F} \circ \pi_{F_{x}, F_{y}} \circ \pi_{F, F_{x}} \mid F_{x}, F_{y} \in T\right\}$. Then $|H|=\left|\Pi_{S^{\prime}}\left(F \cap F^{\prime}\right)\right|$ and $H \leq G^{*}$. By Theorem 11 of [7], $|H|=s+1$ and $\left|G^{*}\right|=s+1$. Hence $H=G^{*}$. As a consequence, $\bar{\theta}$ is an isomorphism if and only if $\theta$ commutes with every element of $G^{*}$.

By the following two theorems, all spreads of symmetry in product and glued near polygons are characterized. The first theorem has been proved in [13] in the case $T \in \tilde{\Upsilon}_{1}(\mathcal{A})$. The result also holds if $T \in \Upsilon_{0}(\mathcal{A})$ (with a similar proof).

Theorem 4.4 (Lemma 7 of [13]). Every admissible spread (spread of symmetry) $S$ of $\mathcal{A}$ is the extension of an admissible spread (spread of symmetry) in $F$ or $F^{\prime}$.

Theorem 4.5. Let $S$ be a spread of symmetry of $F$ and let $G_{S}$ denote the group of automorphisms of $F$ fixing each line of $S$.

(a) If $T \in \Upsilon_{0}(\mathcal{A})$, then $\bar{S}$ is a spread of symmetry of $\mathcal{A}$.

(b) If $T \in \tilde{\Upsilon}_{1}(\mathcal{A})$, let $S^{*}$ denote the spread of $F$ obtained by intersecting $F$ with every element of $T^{C}$ and let $G^{*}$ denote the group of automorphisms of $F$ fixing each line of $S^{*}$. Then $\bar{S}$ is a spread of symmetry of $\mathcal{A}$ if and only if $\left[G^{*}, G_{S}\right]=0$. 


\section{Proof:}

(a) The group $\bar{G}_{S}:=\left\{\bar{\theta} \mid \theta \in G_{S}\right\}$ fixes each line of $\bar{S}$ and acts transitively on each line of $\bar{S}$. So, $\bar{S}$ is a spread of symmetry.

(b) If $\left[G^{*}, G_{S}\right]=0$, then by Proposition $4.3, \bar{G}_{S}:=\left\{\bar{\theta} \mid \theta \in G_{S}\right\}$ is a group of automorphisms of $\mathcal{A}$. Since $\bar{G}_{S}$ fixes each line of $\bar{S}$ and acts transitively on each line of $\bar{S}, \bar{S}$ is a spread of symmetry. Conversely, suppose that $\bar{S}$ is a spread of symmetry, let $G_{\bar{S}}$ denote the group of automorphisms of $\mathcal{A}$ fixing each line of $\bar{S}$. Then $G_{\bar{S}}=\bar{G}$ for some subgroup $G$ of $G_{S}$. By Proposition 11 , we have $\left[G^{*}, G\right]=0$. If $G_{S}=G$, then we are done. If $G_{S} \neq G$, then $\left|G_{S}\right|>|G| \geq s+1$. So, $S$ is a trivial spread of $F$ by the remark following Theorem 9 of [7]. Since $T \in \tilde{\Upsilon}_{1}(\mathcal{A}), S \neq S^{*}$. By Theorem 5 of [7], $S$ and $S^{*}$ have no line in common. By Proposition $4.3(\mathrm{a} 3)$, it then follows that $\left[G^{*}, G_{S}\right]=0$.

\subsection{Compatible spreads of symmetry in product and glued near polygons}

Theorem 4.6. Let $\mathcal{A}$ be a product near polygon, let $\left\{T_{1}, T_{2}\right\} \in \Delta_{0}(\mathcal{A})$, let $F_{1} \in T_{1}$ and let $F_{2} \in T_{2}$. Let $S_{0}$ and $S_{1}$ denote two spreads of symmetry of $F_{1}$ and let $S_{2}$ denote a spread of symmetry of $F_{2}$. Then

(i) $\bar{S}_{1}$ and $\bar{S}_{2}$ are compatible spreads of symmetry of $\mathcal{A}$,

(ii) $\bar{S}_{0}$ and $\bar{S}_{1}$ are compatible spreads of symmetry of $\mathcal{A}$ if and only if $S_{0}$ and $S_{1}$ are compatible spreads of symmetry of $F_{1}$.

Proof: Property (i) follows from Proposition 4.3 (a3). If $\theta_{0} \in G_{0}$ and $\theta_{1} \in G_{1}$, then $\bar{\theta}_{0} \bar{\theta}_{1}=$ $\overline{\theta_{0} \theta_{1}}$ and $\bar{\theta}_{1} \bar{\theta}_{0}=\overline{\theta_{1} \theta_{0}}$. So, $\theta_{0}$ and $\theta_{1}$ commute if and only if $\bar{\theta}_{0}$ and $\bar{\theta}_{1}$ commute. This proves (ii).

Theorem 4.7. Let $\mathcal{A}$ be a glued near polygon, let $\left\{T_{1}, T_{2}\right\} \in \tilde{\Delta}_{1}(\mathcal{A})$, let $F_{1} \in T_{1}$ and let $F_{2} \in T_{2}$. Let $S_{i}^{*}$ denote the spread of symmetry of $F_{i}$ obtained by intersecting $F_{i}$ with the elements of $T_{3-i}$. Let $S_{0}$ and $S_{1}$ denote two spreads of symmetry of $F_{1}$ and let $S_{2}$ denote a spread of symmetry of $F_{2}$. Then

(i) $\bar{S}_{0}$ and $\bar{S}_{1}$ are compatible spreads of symmetry if and only if the spreads $S_{0}, S_{1}$ and $S_{1}^{*}$ are mutually compatible.

(ii) $\bar{S}_{1}$ and $\bar{S}_{2}$ are compatible spreads of symmetry of $\mathcal{A}$ if and only if for every $i \in\{1,2\}, S_{i}$ and $S_{i}^{*}$ are compatible.

\section{Proof:}

(i) We may suppose that the pairs $\left(S_{0}, S_{1}^{*}\right)$ and $\left(S_{1}, S_{1}^{*}\right)$ are compatible (otherwise $\bar{S}_{0}$ and $\bar{S}_{1}$ would not be spreads of symmetry). For every $i \in\{0,1\}$, let $G_{i}$ denote the group of automorphisms of $F_{1}$ fixing each element of $S_{i}$. Since $\left(S_{i}, S_{1}^{*}\right)$ is compatible, $\bar{G}_{i}=$ $\left\{\bar{\theta} \mid \theta \in G_{i}\right\}$ is the full group of automorphisms of $\mathcal{A}$ fixing each element of $\bar{S}_{i}$. If $\theta_{0} \in G_{0}$ and $\theta_{1} \in G_{1}$, then as before $\theta_{0}$ and $\theta_{1}$ commute if and only if $\bar{\theta}_{0}$ and $\bar{\theta}_{1}$ commute. So, $\bar{S}_{0}$ and $\bar{S}_{1}$ are compatible spreads of symmetry of $\mathcal{A}$ if and only if $S_{0}$ and $S_{1}$ are compatible spreads of symmetry of $F$. This proves (i).

(ii) For $\bar{S}_{i}, i \in\{1,2\}$, to be a spread of symmetry it is necessary that $S_{i}$ and $S_{i}^{*}$ are compatible. Conversely, suppose that for every $i \in\{1,2\}, S_{i}$ and $S_{i}^{*}$ are compatible. If $S_{1}=S_{1}^{*}$ and $S_{2}=S_{2}^{*}$, then by (i) it follows that $\bar{S}_{1}=\bar{S}_{2}$ is compatible with itself. We will therefore suppose that $S_{1} \neq S_{1}^{*}$ or $S_{2} \neq S_{2}^{*}$. Then $\bar{S}_{1} \neq \bar{S}_{2}$. Let $L_{1} \in \bar{S}_{1}$ and $L_{2} \in \bar{S}_{2}$ be two lines 
intersecting in a point $x$. Let $U_{i}, i \in\{1,2\}$, denote the unique element of $T_{i}$ through $x$. Since $L_{1} \subseteq U_{1}, L_{2} \subseteq U_{2}, L_{1} \neq L_{2}, \mathcal{C}\left(L_{1}, L_{2}\right)$ is a grid $Q$. By Theorem 5 of [7], the lines of $Q$ disjoint from $L_{i}, i \in\{1,2\}$, belong to $\bar{S}_{i}$. So, the spreads $\bar{S}_{1}$ and $\bar{S}_{2}$ satisfy property (iv) of Theorem 3.1 and hence are compatible. This proves (ii).

\section{Known examples of compatible spreads in dense near polygons}

In this section, we will list all known examples of compatible spreads of symmetry in dense near polygons. By Theorems 4.6 and 4.7, we may content ourselves to those dense near polygons $\mathcal{S}$ for which $\Delta_{0}(\mathcal{S})=\emptyset=\Delta_{1}(\mathcal{S})$.

\subsection{Near polygons with a linear representation}

Let $\mathcal{K}$ be a set of points in $\operatorname{PG}(n, q)$ which satisfies the following properties:

(A) $\langle\mathcal{K}\rangle=\operatorname{PG}(n, q)$,

(B) for every point $x$ of $\mathcal{K}$ and for every line $L$ through $x$, there exists a unique point in $L \backslash\{x\}$ with smallest $\mathcal{K}$-index (the $\mathcal{K}$-index of a point $y$ is the smallest number of points of $\mathcal{K}$ which are necessary to generate a subspace through $y$ ).

Now, embed $\operatorname{PG}(n, q)$ as a hyperplane $\Pi_{\infty}$ in a projective space $\mathrm{PG}(n+1, q)$ and consider the point-line incidence structure $T_{n}^{*}(\mathcal{K})$ whose points are the affine points of $\mathrm{PG}(n+1, q)$ (i.e. the points of $\mathrm{PG}(n+1, q)$ not contained in $\left.\Pi_{\infty}\right)$ and whose lines are the lines of $\mathrm{PG}(n+1, q)$ not contained in $\Pi_{\infty}$ and intersecting $\Pi_{\infty}$ in a point of $\mathcal{K}$ (natural incidence). By Theorem 4.4 of [10], the conditions (A) and (B) imply that $T_{n}^{*}(\mathcal{K})$ is a near polygon. For every point $x$ of $\mathcal{K}$, let $S_{x}$ denote the set of lines of $\operatorname{PG}(n+1, q)$ through $x$ not contained in $\Pi_{\infty}$. The group $H$ of $q$ elations of $\operatorname{PG}(n+1, q)$ with center $x$ and axis $\Pi_{\infty}$ determines a group $G$ of automorphisms of $T_{n}^{*}(\mathcal{H})$. Since $G$ fixes each line of $S_{x}$ and acts regularly on each line of $S_{x}$, the spread $S_{x}$ is a spread of symmetry. By the remark following Theorem 9 of [7], $G$ is the full group of automorphisms of $T_{n}^{*}(\mathcal{K})$ fixing each line of $S_{x}$.

Proposition 5.1 (Theorem 7 of [7]). If $q \geq 3$ (the case of dense near polygons), then every spread of symmetry of $T_{n}^{*}(\mathcal{K})$ is of the form $S_{x}$ for some point $x$ of $\mathcal{K}$.

Since any two elations with axis $\Pi_{\infty}$ commute, we have the following result.

Proposition 5.2. For all $x_{1}, x_{2} \in \mathcal{K}$, the spreads $S_{x_{1}}$ and $S_{x_{2}}$ are compatible.

There are only two examples known of dense near polygons $T_{n}^{*}(\mathcal{K})$ with $\Delta_{0}\left(T_{n}^{*}(\mathcal{K})\right)=$ $\Delta_{1}\left(T_{n}^{*}(\mathcal{K})\right)=\emptyset$. If $\mathcal{K}$ is a hyperoval in $\mathrm{PG}\left(2,2^{h}\right), h \geq 2$, then $T_{2}^{*}(\mathcal{K})$ is a generalized quadrangle of order $\left(2^{h}-1,2^{h}+1\right)$, see [18]. If $\mathcal{K}$ is the Coxeter-cap in $\operatorname{PG}(5,3)([3])$, then $T_{5}^{*}(\mathcal{K})$ is a near hexagon, see [10].

\subsection{The near polygons $H^{D}\left(2 n-1, q^{2}\right), n \geq 2$}

Let the vector space $V\left(2 n, q^{2}\right), n \geq 2$, with base $\left\{\bar{e}_{0}, \ldots, \bar{e}_{2 n-1}\right\}$, be equipped with a nonsingular Hermitian form $(\cdot, \cdot)$, i.e., $\left(\sum a_{i} \bar{e}_{i}, \sum b_{j} \bar{e}_{j}\right)=\sum a_{i} b_{j}^{q}\left(\bar{e}_{i}, \bar{e}_{j}\right)$ for any two vectors $\sum a_{i} \bar{e}_{i}$ and $\sum b_{j} \bar{e}_{j}$ of $V\left(2 n, q^{2}\right)$. Let $\zeta$ denote the corresponding Hermitian polarity of $\mathrm{PG}\left(2 n-1, q^{2}\right)$ and let $H\left(2 n-1, q^{2}\right)$ denote the corresponding Hermitian variety in 
$\operatorname{PG}\left(2 n-1, q^{2}\right)$. The dual polar space $H^{D}\left(2 n-1, q^{2}\right)$ is the point-line incidence structure whose points, respectively lines, are the maximal, respectively next-to-maximal, subspaces of $H\left(2 n-1, q^{2}\right)$. Let $p=\langle\bar{e}\rangle$ denote an arbitrary point of $\operatorname{PG}\left(2 n-1, q^{2}\right)$ not contained in $H\left(2 n-1, q^{2}\right)$. Then $p^{\zeta}$ is a nontangent hyperplane which intersects $H\left(2 n-1, q^{2}\right)$ in a $H\left(2 n-2, q^{2}\right)$. Obviously, the set of all $(n-2)$-dimensional subspaces contained in $p^{\zeta}$ is a spread $S_{p}$ of $H^{D}\left(2 n-1, q^{2}\right)$. For every $\lambda \in \mathrm{GF}\left(q^{2}\right)$ with $\lambda^{q+1}=1$, let $\theta_{\bar{e}, \lambda}$ denote the following linear map of $V\left(2 n, q^{2}\right): \bar{x} \mapsto \bar{x}+(\lambda-1) \frac{(\bar{x}, \bar{e})}{(\bar{e}, \bar{e})} \bar{e}$. The map $\theta_{\bar{e}, \lambda}$ induces an automorphism of PG( $\left.2 n-1, q^{2}\right)$ which fixes the Hermitian variety $H\left(2 n-1, q^{2}\right)$ and every point of the hyperplane $p^{\zeta}$. Since $\theta_{\bar{e}, \lambda_{1}} \circ \theta_{\bar{e}, \lambda_{2}}=\theta_{\bar{e}, \lambda_{1} \lambda_{2}}$ for all $\lambda_{1}, \lambda_{2} \in \mathrm{GF}\left(q^{2}\right)$ with $\lambda_{1}^{q+1}=\lambda_{2}^{q+1}=1$, $H:=\left\{\theta_{\bar{e}, \lambda} \mid \lambda^{q+1}=1\right\}$ is a subgroup of $\operatorname{GL}(2 n, 4)$. Let $G$ denote the group of automorphisms of $H^{D}\left(2 n-1, q^{2}\right)$ induced by the elements of $H$. Then $G$ fixes each line of $S_{p}$. Since $G$ acts regularly on each line of $S_{p}, S_{p}$ is a spread of symmetry of $H^{D}\left(2 n-1, q^{2}\right)$. By the remark following Theorem 9 of [7], $G$ is the full group of automorphisms fixing each line of $S_{p}$. In [12], it was shown that every spread of symmetry is of the form $S_{p}$, where $p$ is a point of PG $\left(2 n-1, q^{2}\right)$ not contained in $H\left(2 n-1, q^{2}\right)$.

Proposition 5.3. Let $p_{1}$ and $p_{2}$ denote two points of $\operatorname{PG}\left(2 n-1, q^{2}\right)$ not contained in $H\left(2 n-1, q^{2}\right)$. Then $S_{p_{1}}$ is compatible with $S_{p_{2}}$ if and only if either $p_{1}=p_{2}$ or $p_{1} \in p_{2}^{\zeta}$.

Proof: Let $\bar{e}_{1}$ and $\bar{e}_{2}$ be vectors of $V\left(2 n, q^{2}\right)$ such that $p_{1}=\left\langle\bar{e}_{1}\right\rangle$ and $p_{2}=\left\langle\bar{e}_{2}\right\rangle$ and let $\lambda_{1}$ and $\lambda_{2}$ denote arbitrary elements of $\operatorname{GF}\left(q^{2}\right) \backslash\{1\}$ satisfying $\lambda_{1}^{q+1}=\lambda_{2}^{q+1}=1$. Then one calculates that $\theta_{\bar{e}_{2}, \lambda_{2}} \circ \theta_{\bar{e}_{1}, \lambda_{1}}$ is the following map:

$$
\bar{x} \mapsto \bar{x}+\left(\lambda_{1}-1\right) \frac{\left(\bar{x}, \bar{e}_{1}\right)}{\left(\bar{e}_{1}, \bar{e}_{1}\right)} \bar{e}_{1}+\left(\lambda_{2}-1\right) \frac{\left(\bar{x}, \bar{e}_{2}\right)}{\left(\bar{e}_{2}, \bar{e}_{2}\right)} \bar{e}_{2}+\left(\lambda_{1}-1\right)\left(\lambda_{2}-1\right) \frac{\left(\bar{x}, \bar{e}_{1}\right)\left(\bar{e}_{1}, \bar{e}_{2}\right)}{\left(\bar{e}_{1}, \bar{e}_{1}\right)\left(\bar{e}_{2}, \bar{e}_{2}\right)} \bar{e}_{2} .
$$

Similarly, one calculates that $\theta_{\bar{e}_{1}, \lambda_{1}} \circ \theta_{\bar{e}_{2}, \lambda_{2}}$ is given by:

$$
\bar{x} \mapsto \bar{x}+\left(\lambda_{1}-1\right) \frac{\left(\bar{x}, \bar{e}_{1}\right)}{\left(\bar{e}_{1}, \bar{e}_{1}\right)} \bar{e}_{1}+\left(\lambda_{2}-1\right) \frac{\left(\bar{x}, \bar{e}_{2}\right)}{\left(\bar{e}_{2}, \bar{e}_{2}\right)} \bar{e}_{2}+\left(\lambda_{1}-1\right)\left(\lambda_{2}-1\right) \frac{\left(\bar{x}, \bar{e}_{2}\right)\left(\bar{e}_{2}, \bar{e}_{1}\right)}{\left(\bar{e}_{1}, \bar{e}_{1}\right)\left(\bar{e}_{2}, \bar{e}_{2}\right)} \bar{e}_{1}
$$

Hence $\theta_{\bar{e}_{1}, \lambda_{1}}$ and $\theta_{\bar{e}_{2}, \lambda_{2}}$ commute if and only if either $\bar{e}_{1} \| \bar{e}_{2}$ or $\left(\bar{e}_{1}, \bar{e}_{2}\right)=0$. The proposition now easily follows.

As a consequence, there are many pairs of compatible spreads of symmetry in $H^{D}(2 n-$ $\left.1, q^{2}\right)$, but not every two spreads of symmetry of $H^{D}\left(2 n-1, q^{2}\right)$ are compatible. In particular, there are many pairs of compatible spreads of symmetry in the GQ $H^{D}\left(3, q^{2}\right) \cong Q(5, q)$.

\subsection{The generalized quadrangle $A S(q), q$ odd}

For every odd prime power $q$, we can construct the following generalized quadrangle $A S(q)$, see [1]. The points of $A S(q)$ are the points of the affine space $A G(3, q)$ and the lines are the following curves in $\mathrm{AG}(3, q)$ (natural incidence):

(1) $x=\sigma, y=a, z=b$;

(2) $x=a, y=\sigma, z=b$;

(3) $x=c \sigma^{2}-b \sigma+a, y=-2 c \sigma, z=\sigma$. 
Here, the parameter $\sigma$ ranges over the elements of $\mathrm{GF}(q)$ and $a, b, c$ are arbitrary elements of $\operatorname{GF}(q)$. The lines of type (1) form a spread $S$.

Proposition 5.4. The spread $S$ is a spread of symmetry which is compatible with itself.

Proof: For every $\lambda \in \mathrm{GF}(q)$, the translation $(x, y, z) \mapsto(x+\lambda, y, z)$ induces an automorphism $\theta_{\lambda}$ of $A S(q)$ which fixes each line of $S$. Obviously, the group $G:=\left\{\theta_{\lambda} \mid \lambda \in \operatorname{GF}(q)\right\}$ acts regularly on every line of $S$. By the remark following Theorem 9 of [7], $G$ is the full group of automorphisms fixing each line of $S$. The proposition now follows from the fact that any two elements of $G$ commute.

If $q \geq 5$, then $S$ is the only spread of symmetry of $A S(q)$ by Theorem 6.3. of [4]. The generalized quadrangle $A S(3) \cong Q(5,2) \cong H^{D}(3,4)$ has more spreads of symmetry as we have seen in Section 5.2.

\subsection{The generalized quadrangle $S_{x, y}^{-}$}

The generalized quadrangle $S_{x, y}^{-}$first occurred in [14], but we give the description taken from [15]. Let $\mathcal{H}$ be a hyperoval in $\mathrm{PG}\left(2,2^{h}\right), h \geq 1$, which is embedded as a hyperplane $\pi$ in $\mathrm{PG}\left(3,2^{h}\right)$ and let $x$ and $y$ be two different points of $\mathcal{H}$. The following generalized quadrangle of order $\left(2^{h}+1,2^{h}-1\right)$ can then be constructed. The points of $S_{x, y}^{-}$are of three types:

(1) points of $\mathrm{PG}\left(3,2^{h}\right)$ not contained in $\pi$;

(2) planes through $x$ not containing $y$;

(3) planes through $y$ not containing $x$.

The lines of $S_{x, y}^{-}$are those lines of $\mathrm{PG}\left(3,2^{h}\right)$ which are not contained in $\pi$ and which intersect $\mathcal{H} \backslash\{x, y\}$. A point of $S_{x, y}^{-}$and a line of $S_{x, y}^{-}$are incident if and only if they are incident as objects of $\mathrm{PG}\left(3,2^{h}\right)$. One easily sees that the points of type (2) form an ovoid $O_{x y}$ of $S_{x, y}^{-}$. Similarly, the points of type (3) form an ovoid $O_{y x}$ of $S_{x, y}^{-}$. [An ovoid (of symmetry) is the dual notion of a spread (of symmetry), i.e., a set of points in a generalized quadrangle $Q$ is called an ovoid (of symmetry) if it is a spread (of symmetry) in the point-line dual of $Q$.]

Proposition 5.5. Each of the pairs $\left(O_{x y}, O_{x y}\right),\left(O_{y x}, O_{y x}\right),\left(O_{x y}, O_{y x}\right)$, is compatible.

Proof: The group $H_{x}$ (respectively $\left.H_{y}\right)$ of the $2^{h}$ elations of $\operatorname{PG}\left(3,2^{h}\right)$ with center $x$ (respectively $y$ ) and axis $\pi$ determine a group $G_{x}$ (respectively $G_{y}$ ) of automorphisms of $S_{x, y}^{-}$. The group $G_{x}$ fixes the ovoid $O_{x y}$ and acts regularly on any set of lines through a given point of $O_{x y}$. This proves that $O_{x y}$ is an ovoid of symmetry. By the remark following Theorem 9 of [7], $G_{x}$ is the full group of automorphisms of $S_{x, y}^{-}$fixing each point of $O_{x y}$. Similarly, $O_{y x}$ is an ovoid of symmetry and $G_{y}$ is the full group of automorphisms of $S_{x, y}^{-}$fixing each point of $O_{y x}$. The proposition now follows from the fact that any two elements of $G_{x} \cup G_{y}$ commute.

If the point-line dual of $S_{x, y}^{-}$is not isomorphic to $T_{2}^{*}(\mathcal{H})$ (see [16] when this precisely occurs), then by [17], $O_{x y}$ and $O_{y x}$ are the only regular ovoids and hence also the only ovoids of symmetry of $S_{x, y}^{-}$. If the point-line dual of $S_{x, y}^{-}$is isomorphic to $T_{2}^{*}(\mathcal{H})$ then there are more than two ovoids of symmetry in $S_{x, y}^{-}$as we have seen in Section 5.1. So, if $h \geq 2$ then 
the generalized quadrangle $\left[S_{x, y}^{-}\right]^{D}$ has either 2 or $q+2$ spreads of symmetry and all these spreads of symmetry are compatible.

\subsection{The near polygons $\mathbb{G}_{n}, n \geq 2$}

Let the vector space $V(2 n, 4), n \geq 2$, with base $\left\{\bar{e}_{0}, \ldots, \bar{e}_{2 n-1}\right\}$ be equipped with the nonsingular Hermitian form $(\bar{x}, \bar{y})=x_{0} y_{0}^{2}+x_{1} y_{1}^{2}+\cdots+x_{2 n-1} y_{2 n-1}^{2}$, let $\zeta$ denote the corresponding Hermitian polarity of $\operatorname{PG}(2 n-1,4)$ and let $H(2 n-1,4)$ denote the corresponding Hermitian variety in $\mathrm{PG}(2 n-1,4)$. The weight of a point $\left\langle x_{0} \bar{e}_{0}+x_{1} \bar{e}_{1}+\cdots+x_{2 n-1} \bar{e}_{2 n-1}\right\rangle$ is defined as the number of $i \in\{0, \ldots, 2 n-1\}$ for which $x_{i} \neq 0$. Let $\mathbb{G}_{n}$ be the incidence structure whose points are the $(n-1)$-dimensional subspaces of $H(2 n-1,4)$ containing precisely $n$ points of weight 2 and whose lines are the $(n-2)$-dimensional subspaces of $H(2 n-1,4)$ containing at least $n-2$ points of weight 2 (incidence is reverse containment). It was shown in [8] that $\mathbb{G}_{n}$ is a dense near polygon of order $\left(2, \frac{3 n^{2}-n-2}{2}\right)$. For every $i \in\{0, \ldots, 2 n-1\}$, let $S_{i}$ denote the set of $(n-2)$-dimensional subspaces of $H(2 n-1,4)$ which are contained in $\left\langle\bar{e}_{i}\right\rangle^{\zeta}$ and which contain at least $n-2$ points of weight 2 . By Lemma 15 of [8], $S_{i}$ is a spread of symmetry of $\mathbb{G}_{n}$.

Proposition 5.6. For all $i, j \in\{0,1, \ldots, 2 n-1\}, S_{i}$ is compatible with $S_{j}$.

Proof: For every $\lambda \in \mathrm{GF}(4)^{*}$ and every $i \in\{0, \ldots, 2 n-1\}$, let $\theta_{i, \lambda}$ denote the following linear map of $V(2 n, 4): \bar{e}_{i} \mapsto \lambda \bar{e}_{i}$ and $\bar{e}_{j} \mapsto \bar{e}_{j}$ for every $j \in\{0, \ldots, 2 n-1\} \backslash\{i\}$. Obviously, the linear map $\theta_{i, \lambda}$ induces an automorphism $\tilde{\theta}_{i, \lambda}$ of $\mathbb{G}_{n}$. Now, let $G_{i}, i \in\{0, \ldots, 2 n-1\}$, denote the group of automorphisms of $\mathbb{G}_{n}$ fixing each line of $S_{i}$. By the remark following Theorem 9 of [7], we know that $G_{i}:=\left\{\tilde{\theta}_{i, \lambda} \mid \lambda \in \mathrm{GF}(4)^{*}\right\}$. It is now easy to see that $\left[G_{i}, G_{j}\right]=0$ for all $i, j \in\{0, \ldots, 2 n-1\}$.

If $n \geq 3$, then the spreads $S_{i}, i \in\{0, \ldots, 2 n-1\}$, are the only spreads of symmetry of $\mathbb{G}_{n}$ (Corollary 3 of [8]). As a consequence, $\mathbb{G}_{n}, n \geq 3$, has $2 n$ spreads of symmetry and all these spreads are mutually compatible. The generalized quadrangle $\mathbb{G}_{2} \cong Q(5,2) \cong H^{D}(3,4)$ has more than $2 n=4$ spreads of symmetry and not every pair of such spreads is compatible, as we have seen in Section 5.2.

\section{References}

1. R.W. Ahrens and G. Szekeres, "On a combinatorial generalization of 27 lines associated with a cubic surface," J. Austral. Math. Soc. 10 (1969), 485-492.

2. A.E. Brouwer and H.A. Wilbrink, "The structure of near polygons with quads," Geom. Dedicata 14 (1983), $145-176$.

3. H.S.M. Coxeter, "Twelve points in PG(5, 3) with 95040 self-transformations," Proc. Roy. Soc. London Ser. A 247 (1958) 279-293.

4. B. De Bruyn, "Generalized quadrangles with a spread of symmetry," European J. Combin. 20 (1999) 759-771.

5. B. De Bruyn, "On near hexagons and spreads of generalized quadrangles," J. Algebraic Combin. 11 (2000), 211-226.

6. B. De Bruyn, "Glued near polygons,” Europ. J. Combin. 22 (2001) 973-981.

7. B. De Bruyn, "The glueing of near polygons," Bull. Belg. Math. Soc. Simon Stevin 9 (2002) 610-630.

8. B. De Bruyn, "New near polygons from hermitean varieties," Bull. Belg. Math. Soc. Simon Stevin 10 (2003), 561-577.

9. B. De Bruyn, "Decomposable near polygons,” Ann. Combin. 8 (2004) 251-267. 
10. B. De Bruyn and F. De Clerck, "On linear representations of near hexagons," Europ. J. Combin. 20 (1999) 45-60.

11. B. De Bruyn and P. Vandecasteele, "Two conjectures regarding dense near polygons with three points per line," Europ. J. Combin. 24 (2003) 631-647.

12. B. De Bruyn and P. Vandecasteele, "Near polygons having a big sub near polygon isomorphic to $H^{D}(2 n-$ 1, 4)," To appear in Ars Combin.

13. B. De Bruyn and P. Vandecasteele, "Near polygons with a nice chain of sub near polygons," J. Combin. Theory Ser. A 108 (2004) 297-311.

14. S.E. Payne, "Nonisomorphic generalized quadrangles," J. Algebra 18 (1971) 201-212.

15. S.E. Payne, "Hyperovals and generalized quadrangles," in C.A. Baker and L.A. Batten (eds), Finite Geometries, volume 103 of Lecture Notes in Pure and Applied Mathematics, M. Dekker, 1985 pp. 251271.

16. S.E. Payne, "Hyperovals yield many GQ," Simon Stevin 60 (1986) 211-225.

17. S.E. Payne and M.A. Miller, "Collineations and characterizations of generalized quadrangles of order $(q-1, q+1)$, , Supplimenti ai Rendiconti del circolo Matematico di Palermo 53(II) (1998) 137-166.

18. S.E. Payne and J.A. Thas, Finite Generalized Quadrangles, volume 110 of Research Notes in Mathematics. Pitman, Boston, 1984.

19. E.E. Shult and A. Yanushka, "Near $n$-gons and line systems," Geom. Dedicata 9 (1980) 1-72.

20. H. Van Maldeghem. Generalized Polygons, volume 93 of Monographs in Mathematics. Birkhäuser, Basel, Boston, Berlin, 1998. 